\title{
On the Application of Proper Orthogonal Decomposition (POD) for In-Cylinder Flow Analysis
}

\author{
Mohammed El-Adawy ${ }^{1}$ (D), Morgan R. Heikal ${ }^{1,2}$, A. Rashid A. Aziz ${ }^{1, *}$, Ibrahim Khalil Adam ${ }^{1}$, \\ Mhadi A. Ismael ${ }^{1}$, Mohammed E. Babiker ${ }^{1}$, Masri B. Baharom ${ }^{1}$, Firmansyah ${ }^{1}$ (i) \\ and Ezrann Zharif Zainal Abidin ${ }^{1}$ \\ 1 Centre for Automotive Research and Electric Mobility, Mechanical Engineering Department, \\ Universiti Teknologi PETRONAS 32610, Seri Iskandar, Perak, Malaysia; \\ engmohammed_2008@yahoo.com (M.E.-A.); M.R.Heikal@brighton.ac.uk (M.R.H.); \\ himakh80@gmail.com (I.K.A.); xmhadosx@yahoo.com (M.A.I.); mohammedelamen@gmail.com (M.E.B.); \\ masrib@utp.edu.my (M.B.B.); firmansyah@utp.edu.my (F.); ezrann.zainal@utp.edu.my (E.Z.Z.A.) \\ 2 The Advanced Engineering Centre based at the Sir Harry Ricardo Laboratories, University of Brighton, \\ Brighton BN2 4GJ, UK \\ * Correspondence: kotb_g03443@utp.edu.my; Tel.: +60-183537595
}

Received: 10 July 2018; Accepted: 18 August 2018; Published: 28 August 2018

\begin{abstract}
Proper orthogonal decomposition (POD) is a coherent structure identification technique based on either measured or computed data sets. Recently, POD has been adopted for the analysis of the in-cylinder flows inside internal combustion engines. In this study, stereoscopic particle image velocimetry (Stereo-PIV) measurements were carried out at the central vertical tumble plane inside an engine cylinder to acquire the velocity vector fields for the in-cylinder flow under different experimental conditions. Afterwards, the POD analysis were performed firstly on synthetic velocity vector fields with known characteristics in order to extract some fundamental properties of the POD technique. These data were used to reveal how the physical properties of coherent structures were captured and distributed among the POD modes, in addition to illustrate the difference between subtracting and non-subtracting the ensemble average prior to conducting POD on datasets. Moreover, two case studies for the in-cylinder flow at different valve lifts and different pressure differences across the air intake valves were presented and discussed as the effect of both valve lifts and pressure difference have not been investigated before using phase-invariant POD analysis. The results demonstrated that for repeatable flow pattern, only the first mode was sufficient to reconstruct the physical properties of the flow. Furthermore, POD analysis confirmed the negligible effect of pressure difference and subsequently the effect of engine speed on flow structures.
\end{abstract}

Keywords: in-cylinder flow; coherent structures; POD; PIV

\section{Introduction}

The in-cylinder flow characteristics are proven to have a significant impact on the performance, combustion and emissions of an internal combustion engine [1,2]. Therefore, studying the in-cylinder flow structures during the intake and compression strokes remains a subject of great interest in the automotive research community. In-cylinder flow structures generated during the intake stroke and modified during the compression stroke can be divided into two main forms of large scale motions (coherent structures), swirl and tumble. Swirl is the in-cylinder flow where the flow rotational axis is parallel to the cylinder axis while tumble is the flow with an axis perpendicular to that of the cylinder [3-5]. These coherent structures are mainly relying on the bore/stroke ratio, intake valve geometry, inlet port profile and the shape of the combustion chamber [6-9]. However, with the 
advent of more sophisticated mixture flow strategies in engines to meet the recent emission standards, the understanding of in-cylinder flow characteristics became more than ever a challenge for the research community. In addition, there is the problem of how to distinguish between turbulence and cycle to cycle variability. Therefore, in the recent years, the proper orthogonal decomposition analytical technique introduced firstly by Lumley [10] to the turbulence research community is considered as a powerful method to extract the most energetic structures in turbulent flows. The applications of POD have seen an increase in engine flow studies, in-cylinder flow analysis $[4,11,12]$ cycle to cycle variations [13-18], variation of fuel spray structure $[19,20]$ and combustion instability and misfire [14,21]. The aforementioned studies mainly have been concentrated on the evaluation of cycle-to-cycle variability in engines, either for air flow or for spray flow using POD. Two main POD approaches can be used to process the in-cylinder turbulent flow datasets, which are "phase-dependent POD" and "phase-invariant POD". The phase-dependent POD approach is to implement the POD at each phase of the flow separately. Whilst, on the other hand, the phase-invariant POD approach is to perform the POD over all available phases of the flow at the same time [22].

In the current study, Stereoscopic PIV measurements were carried out at the central vertical tumble plane inside an engine cylinder to acquire the instantaneous velocity vector fields of the in-cylinder flow for two experimental conditions, i.e., (1) different valve lift, and (2) different pressure difference across the air intake valves. Here, as also used by Kapitza et al. [23] and Hao Chen et al. [15,21,24], the instantaneous velocity vector fields acquired from Stereo-PIV measurements were arranged firstly in the form of synthetic velocity fields in order to extract some fundamental properties of the POD technique (avoiding the complexities of real flow). These data were used to reveal how the physical properties of coherent structures were captured and distributed among the POD modes, in addition to illustrate the difference between subtracting and non-subtracting the ensemble average prior to conducting POD on datasets. Moreover, phase-invariant POD was then implemented to evaluate the effect of valve lift and pressure difference on the in-cylinder flow variation and evolution. The combination of the steady-state flow rig as a qualitative tool, the high-speed stereoscopic particle image velocimetry and the powerful statistical analysis technique proper orthogonal decomposition (POD) as quantitative tools can thus offer a new insight into in-cylinder flows and can be utilized as an engine design tool. Therefore, the objective of this work is to gain more understanding of the applications of POD technique to the in-cylinder flows of IC engines through samples of synthetic velocity vector fields and two real case studies.

\section{Experimental Set Up}

An Forschungsgesellschaft für Energietechnik und Verbrennungsmotoren (FEV) steady-state flow rig was adopted to apply stereoscopic PIV measurements at different valve lifts. The experimental set up can be divided into two main parts, steady-state flow rig and stereoscopic PIV system as shown in Figure 1.

\subsection{Steady-State Flow Bench}

The steady-state flow rig consisted of a centrifugal compressor working under suction conditions. A gasoline direct injection (GDI) engine head was mounted on the flow rig while the air was inducted through the intake ports, intake valves, and optical cylinder then finally discharged to the atmosphere to simulate the engine intake stroke. The pressure difference adjustment across the intake valves was achieved using a by-bass controlled by a steeper motor while the air flow rate was measured using rotary piston flow meter. 


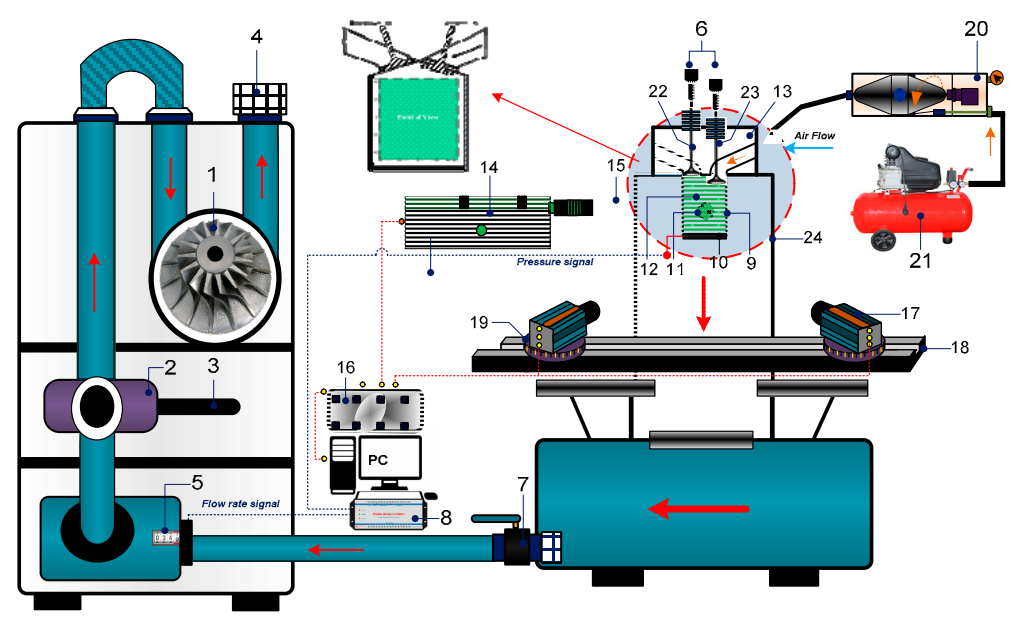

\begin{tabular}{|c|c|c|}
\hline 1-Centrifugal compressor & 9- Optical cylinder & 17-CCD-camera \\
\hline 2-By pass & 10- Flat piston crown & 18-Traverse system \\
\hline 3-Steeper motor & 11-Air outlet ports & 19-Scheimpflug camera mount \\
\hline 4-Air filter & 12- Area of interest & 20-Powder seeding generator \\
\hline 5- Rotary piston flow meter & 13-Cylinder head & 21-Air compressor \\
\hline 6-Micrometer & 14-Laser generator & 22- Exhaust valves \\
\hline 7-Stop cock & 15-Laser sheet & 23-Intake valves \\
\hline 8-Data acquisition system & 16-Timer box & 24-Correction box \\
\hline
\end{tabular}

Figure 1. Experimental setup showing the field of view.

\subsection{The Stereoscopic PIV Setup}

The principle of stereoscopic PIV technique is to record the flow with two synchronized cameras from different view angles $[25,26]$. The three components of the velocity vectors can then directly be obtained from the two measured vector fields. In the current study, a high speed stereoscopic PIV (2D3C-Dantec Dynamics, Skovlunde, Denmark) was used through the mid-cylinder tumble-plane (plane of symmetry) at different valve lifts and different pressure differences across the air intake valves. The acquired velocity vector fields were used as input datasets to perform the POD analysis. Mainly the experimental set-up of the stereo PIV consists of the following elements, pulsed laser system, two CCD-cameras, two stereo (Scheimpflug) camera mounts, calibration targets and analysis software $[27,28]$. With PIV, the velocity of particles suspended in the flow of interest is measured-i.e., seeding particles are used as "velocity probes". Therefore, the precise choice of seeding particles is critical to the successful implementation of PIV experiments. During this study, titanium dioxide $\left(\mathrm{TiO}_{2}\right)$ was used as seeding particles and generated by means of solid particle seeder and mixed with air through the intake ports. Moreover, as PIV measurements rely on micro sized tracer particles suspended in the flow, subsequently, it is necessary to use a high power light source in order to ensure sufficient scattering light from these particles which can be captured by the CCD cameras. The light source used for this study was two CW Q-switched Nd: YLF DPSS laser resonators (Dual Power 65-15) (Litron Lasers, Rugby, UK)) producing infrared laser light at $1053 \mathrm{~nm}$ which is converted to visible $527 \mathrm{~nm}$ laser light by an intra-cavity Harmonic Generation Assembly (HGA). The harmonic generator produced a laser output at $527 \mathrm{~nm}$ (green). The Nd: YLF double pulsed laser is capable of $20 \mathrm{~mJ}$ at $1 \mathrm{kHz}$ at $527 \mathrm{~nm}$ per laser head per pulse. A pair of Speed Sense M310 Dantec Dynamics cameras running in double frame mode were used. A point of particular importance is the choice of the aperture used to capture the images. The aperture controls the amount of light striking the camera sensor. As a consequence, it affects both the luminosity of image and the depth of focus. The larger the aperture, the smaller the depth of focus, the higher the luminosity of the picture [29]. It was adjusted at 8 in order to allow enough amount of light to pass through and achieve appropriate depth of focus. The calibration was done by means of a standard calibration target which had a well-defined grid of dots. The standard calibration target was one-sided plate containing black dots on a white background 
spacing of $5 \mathrm{~mm}$ and a size of $100 \mathrm{~mm}$ by $100 \mathrm{~mm}$. The final PIV images (1280 pixels by 800 pixels, 12-bit grey scale) were saved directly onto the hard drive of the computer for analysis. The data analysis was accomplished using adaptive correlation with an interrogation area size of $32 \times 32$ pixels and $50 \%$ overlapping. The Scheimpflug principle is a geometric rule that describes the orientation of the plane of focus of camera when the lens plane is not parallel to the image plane (When looking at the light sheet at an angle instead of head on, the camera has to be tilted with respect to the lens) as illustrated in Figure 2. To fulfil the Scheimpflug condition and ensure proper focusing (which requires that the object plane, the lens plane and the image plane are collinear) a special camera mount was required. This mount made it possible to tilt the image-plane (CCD-plane) relative to the lens-plane around $\mathrm{CCD}$ axis. After generating the raw velocity fields, a moving average validation and an average filter techniques were applied to deal with the spurious vectors.

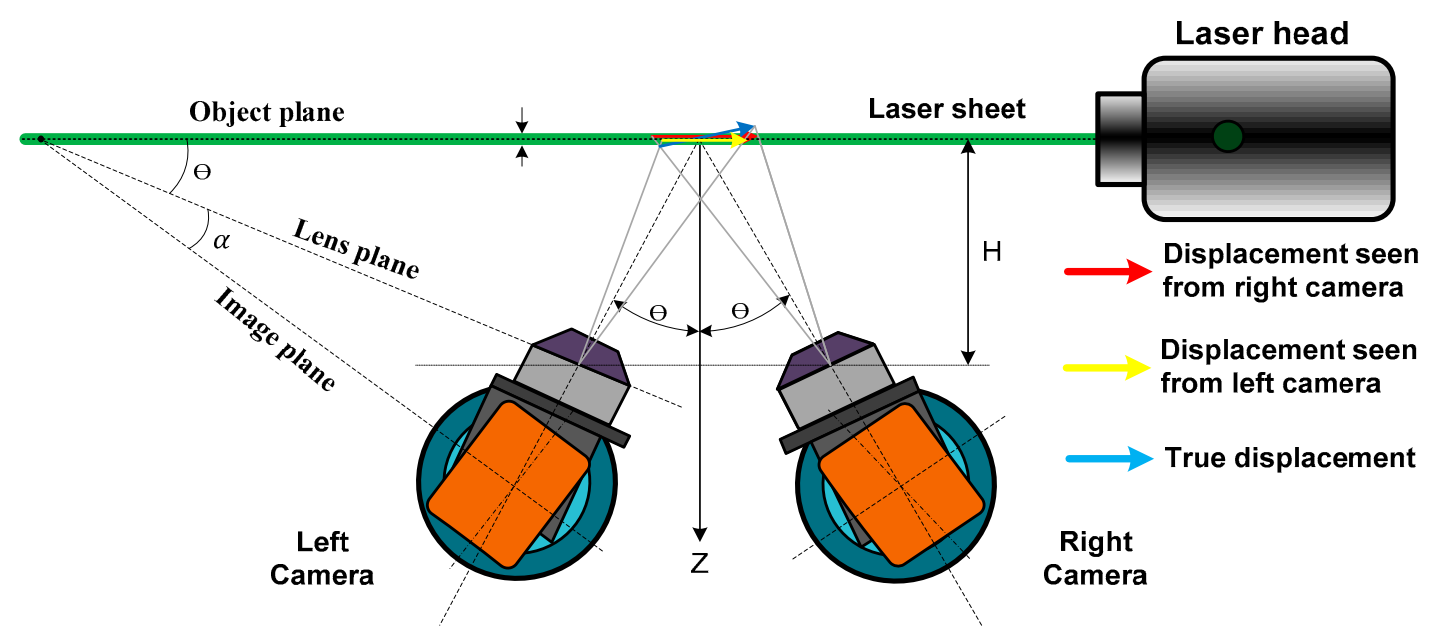

Figure 2. Scheimpflug stereoscopic camera configuration.

\section{D Stereoscopic PIV Data Processing}

This section gives a brief description of the processing required for computing 2D3C stereoscopic PIV vector maps, as shown in Figure 3, which can be summarized as follows:

- The two camera views were calibrated by recording images of a standard calibration target

- Image Model Fit (IMF) calibration files for camera 1 and camera 2 were calculated using the Dantec Dynamic software

- Stereoscopic-measurements started with conventional 2D-PIV processing of PIV images recorded simultaneous flow field as seen from the cameras (i.e., not correlated by the angular view)

- Using the camera view calibrations calculated earlier (IMF), these two 2D vector maps from both cameras were then combined into one single 3D vector map using the stereoscopic PIV processing method. 


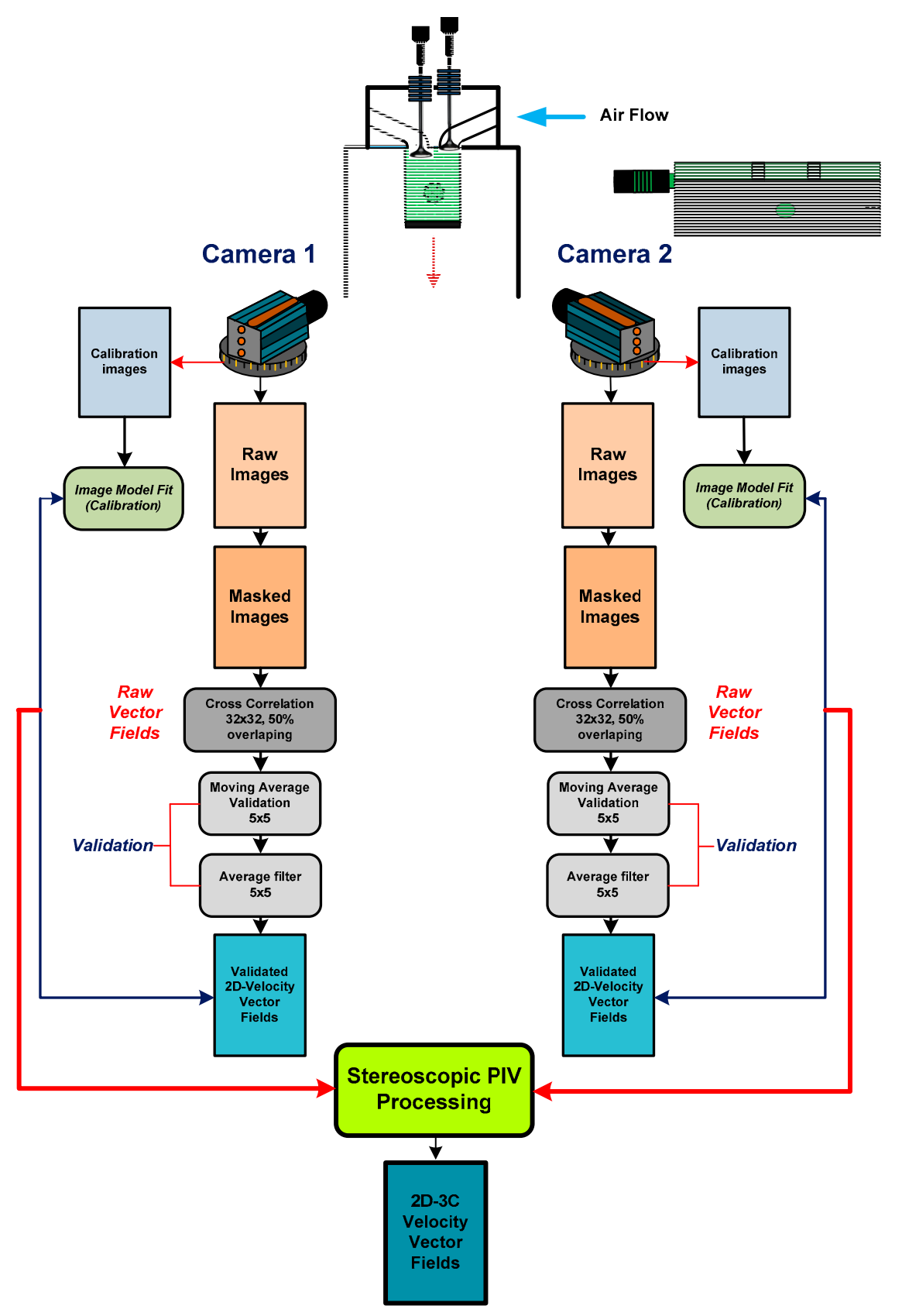

Figure 3. Flow chart representing vectors calculation process for stereoscopic PIV.

\section{Proper Orthogonal Decomposition}

The POD decomposes a set of velocity distributions (snapshots) with total number of $(K)$ into a linear combination of $(M)$ spatial basis functions (or space-dependent POD modes, (denoted $\varphi_{m}$ ) and their corresponding time-dependent coefficients, (denoted $C_{m}^{(k)}$ ). A brief description of the mathematical procedure of the method of snapshot proposed by Sirovich [30] can be presented as follows:

$$
(V)^{(k)}=\sum_{m=1}^{M} C_{m}^{(k)} \times \varphi_{m}
$$

The POD code in previous paper [4] was employed here. The code mathematically minimizes the following:

$$
\sum_{k=1}^{k}\left\|(V)^{(k)}-\sum_{m=1}^{M} C_{m}^{(k)} \times \varphi_{m}\right\|^{2} \rightarrow \min
$$


Since $\varphi_{m}$ is normalized and $C_{m}^{(k)}$ is the amplitude, $\frac{1}{2}\left(C_{m}^{(k)}\right)^{2}$ quantifies the kinetic energy $(K E)$ per unit mass the $m$ th mode contributes in the $k$ th velocity field.

$$
K E_{m}=\frac{1}{2}\left(C_{m}^{(k)}\right)^{2}
$$

\section{The Calculation Process in Detail}

Consider a set of velocity fields to be processed: $V^{(k)}=(u, v)_{i, j}^{(k)},(k=1,2,3, \cdots \cdots, K)$. Here, $i, j$ are the spatial position coordinates of the velocity vector in the velocity field, $k$ the velocity field index and $u, v$ are the velocity components in $\mathrm{x}$ and $\mathrm{y}$ directions respectively. Organize a total of $\mathrm{I} \times \mathrm{J}$ velocity vectors in all $K$ velocity fields and each velocity field is collated as follows:

$$
U=\left[\begin{array}{c}
U^{(1)} \\
U^{(2)} \\
\vdots \\
U^{(K)}
\end{array}\right]=\left[\begin{array}{ccccccc}
u_{i=1, j=1}^{(1)} & u_{i=1, j=2}^{(1)} & \cdots & u_{i=1, j=J}^{(1)} & u_{i=2, j=1}^{(1)} & \cdots & u_{i=I, j=J}^{(1)} \\
u_{i=1, j=1}^{(2)} & u_{i=1, j=2}^{(2)} & \cdots & u_{i=1, j=J}^{(2)} & u_{i=2, j=1}^{(2)} & \cdots & u_{i=I, j=J}^{(2)} \\
\cdots & \cdots & \cdots & \cdots & \cdots & \cdots & \cdots \\
u_{i=1, j=1}^{(K)} & u_{i=1, j=2}^{(K)} & \cdots & u_{i=1, j=J}^{(K)} & u_{i=2, j=1}^{(K)} & \cdots & u_{i=I, j=J}^{(K)}
\end{array}\right]
$$

The y-direction velocity is made to be the same as the Equation (10), and the matrix V is obtained. Then, define the spatial correlation matrix as follows:

$$
C=\frac{1}{K}\left(U U^{T}+V V^{T}\right)
$$

As mentioned in the previous section, in order to solve the minimization problem described in (2), the eigenvalue problem of the spatial correlation matrix needs to be solved, namely:

$$
C \beta_{m}=\lambda_{m} \beta_{m}
$$

where $\lambda_{m}$ is the eigenvalue of the spatial correlation matrix of the input flow field set, and $\beta_{m}$ is its eigenvector.

Each eigenvalue corresponds to a characteristic vector. The larger the eigenvalue, the longer the corresponding coordinate axis, and the stronger its dominance. are arranged in descending order according to the magnitude of eigenvalues as:

$$
\lambda^{1}>\lambda^{2}>\lambda^{3} \ldots \ldots>\lambda^{K}=0
$$

The POD coefficients $\left(C_{m}^{(k)}\right)$ are obtained by projecting the input velocity field $V^{(k)}$ onto the basis function $\varphi_{m}$. Finally get a $\mathrm{K} \times \mathrm{M}$ POD coefficient matrix:

$$
C_{m}^{(k)}=\left[\begin{array}{cccc}
C_{1}^{(1)} & C_{2}^{(1)} & \cdots & C_{M}^{(1)} \\
C_{1}^{(2)} & C_{2}^{(2)} & \cdots & C_{M}^{(2)} \\
\vdots & \vdots & \vdots & \vdots \\
C_{1}^{(K)} & C_{2}^{(K)} & \cdots & C_{M}^{(K)}
\end{array}\right]
$$

The coefficient $C_{m}^{(k)}$ represents the strength of the input flow field $V^{(k)}$ in the flow field structure of the basis function $\varphi_{m}$. As mentioned earlier, the basis functions are sorted in descending order of energy, that is, the first basis function contains the highest share of energy, and the second basis function contains the second highest share of energy. Figure 4 summarized the main principle of POD. 


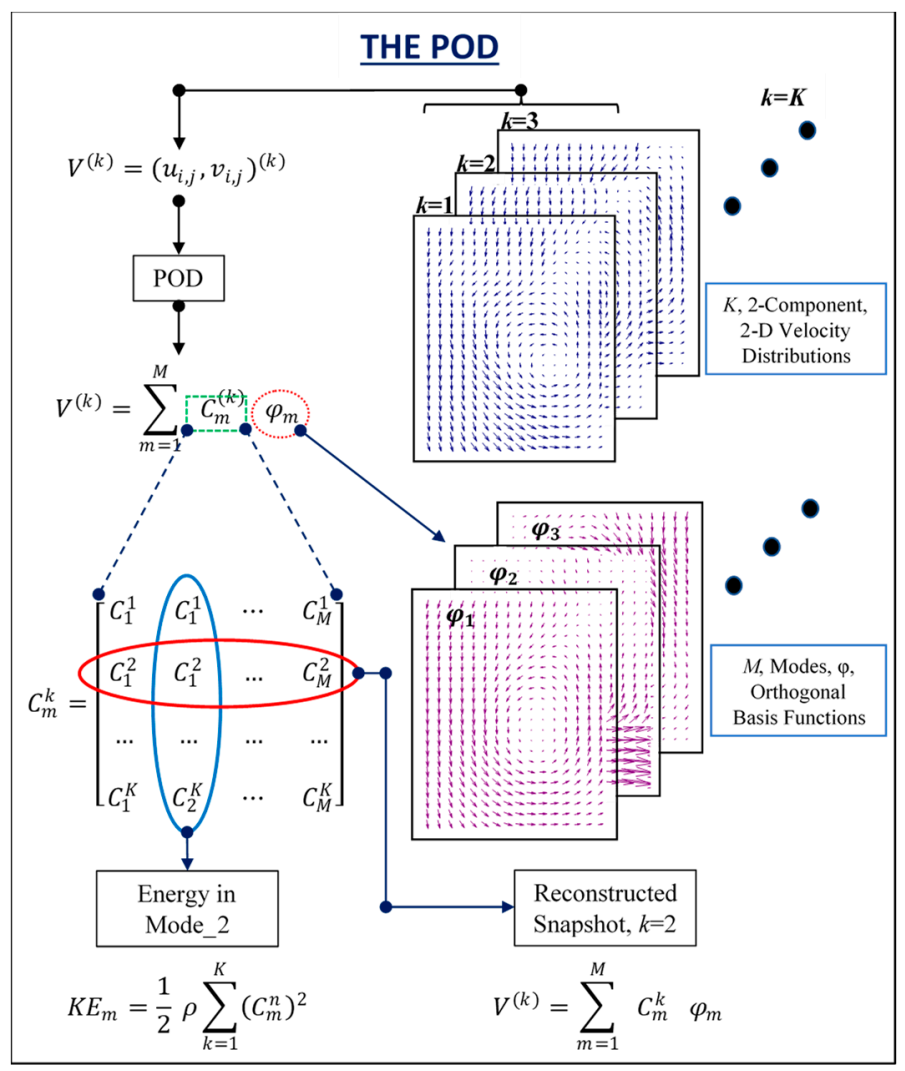

Figure 4. Schematic showing the main principle of POD.

\section{Results}

\subsection{POD Implementation of Repeated Identical Flow Patterns}

Figure 5 shows how the energy of identically repeated flow patterns was captured and distributed among the POD modes (or basis functions). One velocity vector field, showing the counterclockwise tumble motion at valve lift $10 \mathrm{~mm}$, was repeated in 100 snapshots. Consequently, 100 modes resulted without subtracting the ensemble average prior to performing the POD on the data set. This example demonstrated two main properties of POD. Firstly, it is well known that each POD mode comprises elements of all flow structures from all input snapshots therefore for this example mode $1(\varphi 1)$ was enough to capture the same structures as the original snapshots having $99.9 \%$ of total energy while the other modes contained just noise. It could be concluded also that only the first mode was adequate to reconstruct the physical flow structures. Secondly, as the same structure was repeated in all snapshots, the magnitude of coefficients for specific POD mode which illustrated to what extent this mode was dominant for a particular snapshot was distributed equally between all snapshots, as shown in Figure 6. For mode 1 coefficients, the values were identical and all positive, meaning that the mode 1 was dominant in all snapshots and the positive values indicated that structures captured by mode 1 were in the same direction as the original snapshots. For mode 2 coefficients, they were all also similar but negative values meaning that structures captured by mode 2 were in the opposite direction compared to the original snapshots. 


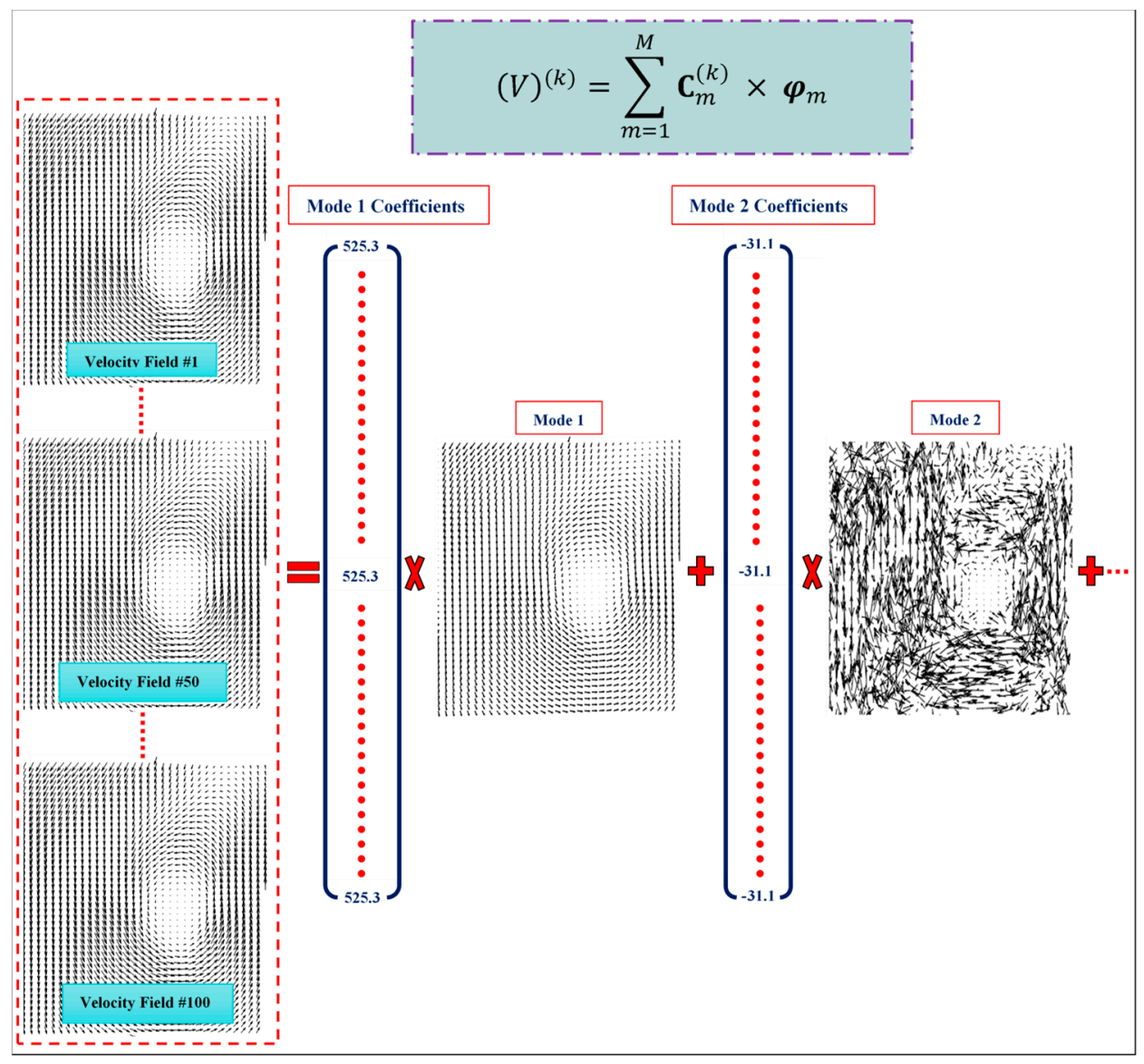

Figure 5. Illustration of POD for repeated identical flow structures.



Figure 6. Coefficients of the first two POD modes for all 100 input velocity vector fields.

\subsection{Capturing the Mechanism of Coherent Structures}

Figure 7 reveals how coherent structures were captured and distributed among the POD modes. One velocity vector field contained the weakest structures, showing the reverse tumble motion in the clock wise direction, was repeated at same location and with the same orientation in 50 snapshots which had a total kinetic energy of 2,003,133 $\mathrm{m}^{2} / \mathrm{s}^{2}\left(40,062.67 \mathrm{~m}^{2} / \mathrm{s}^{2}\right.$ per one velocity field). Another velocity vector field contained the most energetic structures, showing a strong tumble motion in the counter 
clock-wise direction, was repeated at same location and with the same orientation in 50 snapshots which had a total kinetic energy of $23,368,755 \mathrm{~m}^{2} / \mathrm{s}^{2}\left(467,375.1 \mathrm{~m}^{2} / \mathrm{s}^{2}\right.$ per velocity field). Consequently, 100 snapshots were created as the input velocity vector fields and resulted in 100 modes without subtracting the ensemble average prior to performing the POD on the data set. As can be seen from the figure, mode $1\left(\varphi_{1}\right)$ captured only the structures which had the maximum kinetic energy found in the last 50 velocity fields $V^{(51-100)}$ comprising $92.5 \%$ of total energy. Then mode $2\left(\varphi_{2}\right)$ captured the structures found in the first 50 snapshots $V^{(1-50)}$ which had the lowest kinetic energy comprising $7.3 \%$ of total energy. The coefficients of the first two POD modes for all snapshots are listed in Figure 8, which reveals the magnitude and sign by which each mode was scaled. The coefficients of the first mode $C_{1}^{(k=51-100)}$ were similar, positive and large for all $\mathrm{k}$ since $V^{(51-100)}$ contained the strongest structures and almost all their energies were captured by mode $1\left(\varphi_{1}\right)$. While on the other hand, the coefficients of the second mode $C_{2}^{(k=51-100)}$ were almost zero since they did not exist in $V^{(51-100)}$. Similar insight could be gained by analysing the behaviour of the coefficients of the second mode $C_{2}^{(k=1-50)}$. This example also demonstrated an important feature of POD about the snapshot-to-snapshot difference which could be captured by the corresponding coefficients. This principle was considered as a key factor for the quantitative study of cycle-to-cycle variation in real engine flows.

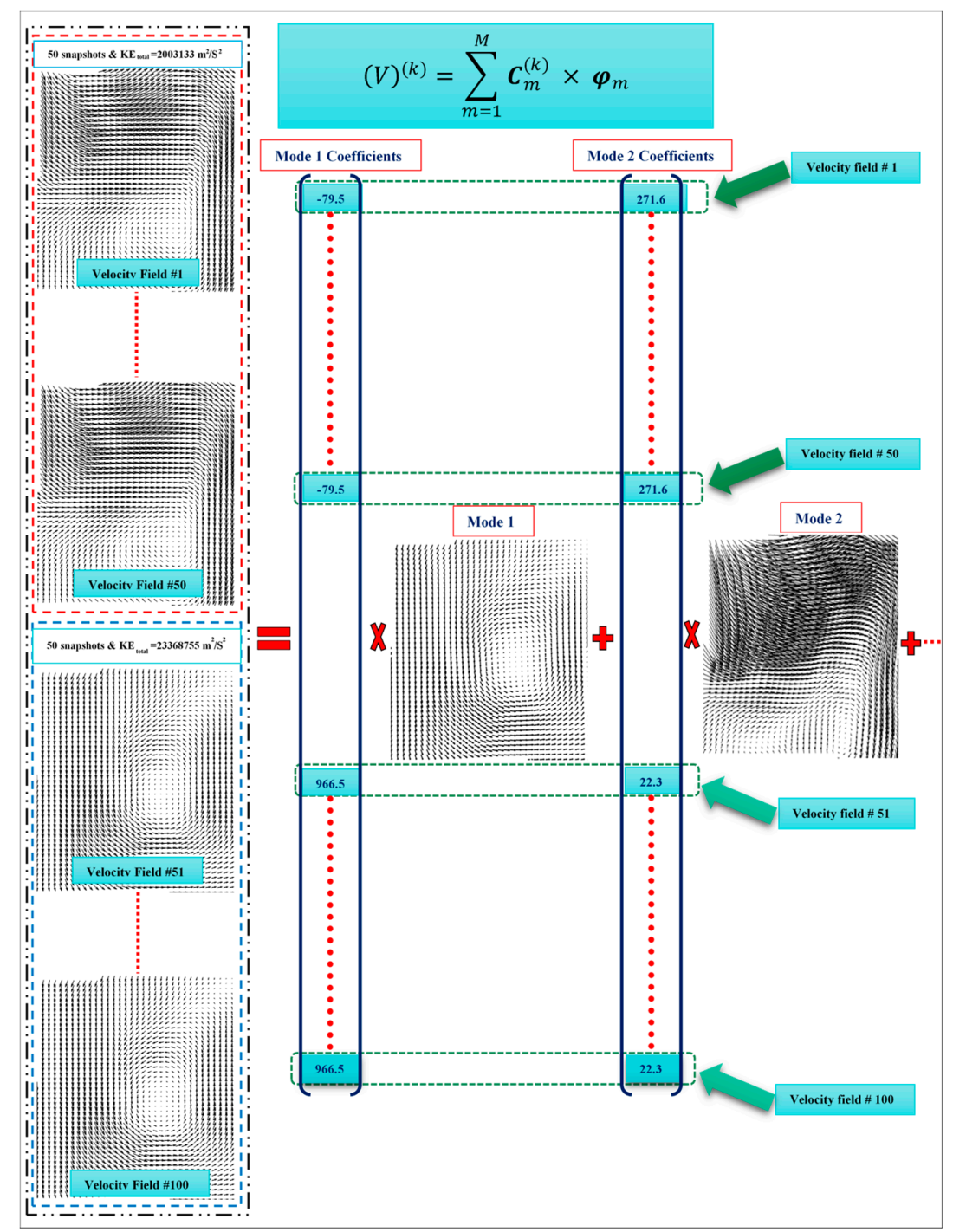

Figure 7. Illustration of POD for capturing of coherent structures. 


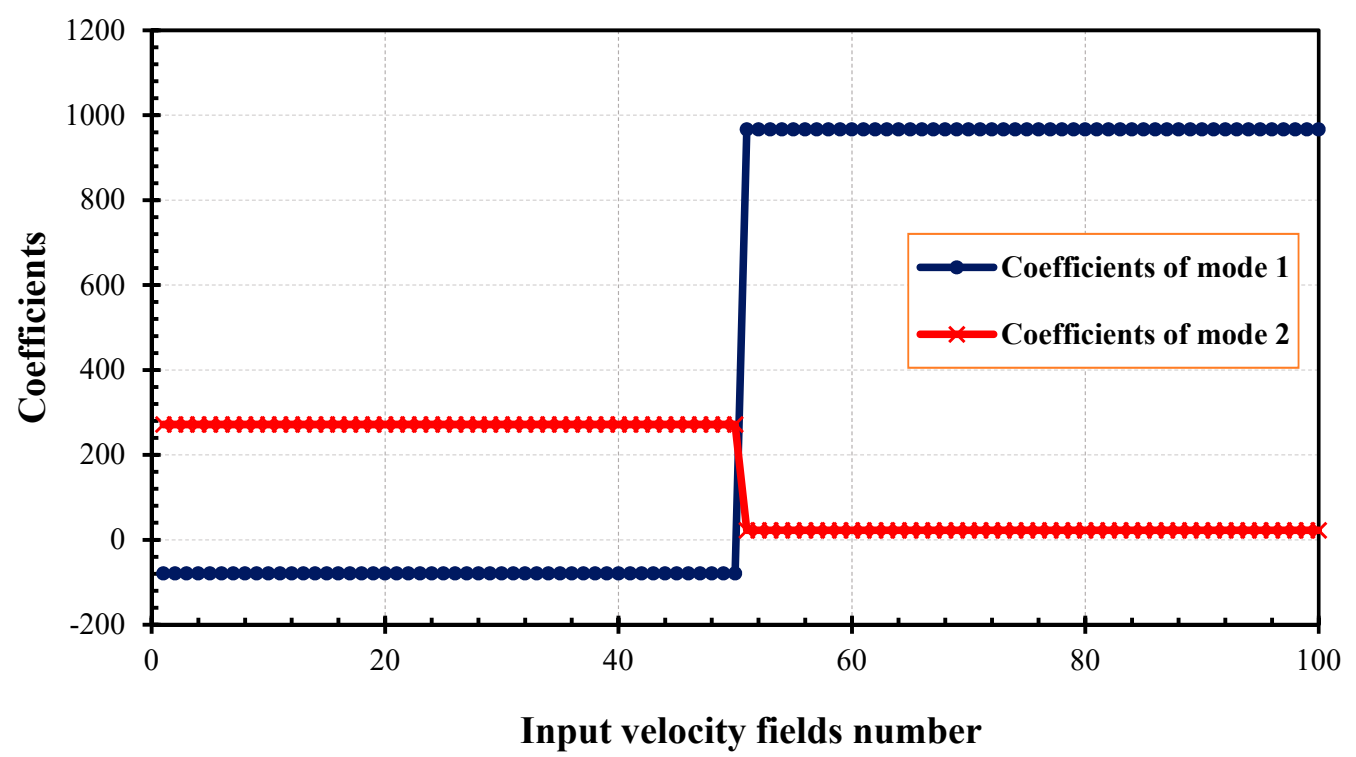

Figure 8. Coefficients of first two POD modes for all 100 input velocity vector fields.

\subsection{POD and the RANS Turbulence (Subtracting and Non-Subtracting the Ensemble Average Prior to} Applying POD on the Dataset)

The aim of the current example was to reveal to what extent subtracting or not subtracting the ensemble average prior to performing the POD analysis on a data set was effective. This included a complete comparison between flow patterns of the POD modes derived with and without subtracting the ensemble average. In this example, a 200 instantaneous velocity vector fields $(K=200)$ at valve lift $9 \mathrm{~mm}$ and $150 \mathrm{~mm} \mathrm{H}_{2} \mathrm{O}$ pressure difference were used to reveal the physical properties of the POD modes in this case.

\subsubsection{The Ensemble Average and the First POD Mode Relationship}

Figure 9 compares the spatial structures of both mode $1\left(\varphi_{1}\right)$ created from the original velocity vector fields, $V$, and the ensemble average, $\langle\mathrm{V}\rangle$. It can be noticed that the flow pattern of the first mode was an excellent estimate of the flow pattern of the ensemble average and had the same direction. This gave an indication that the coefficients would be positive. The kinetic energy content of both the ensemble average and POD mode 1 are compared in Table 1 . Mode 1 kinetic energy $\left(\mathrm{KE}_{1}\right)$ was calculated from the coefficients $\left(\frac{1}{2}\left(C_{1}^{(k)}\right)^{2}\right)$ and divided by $K=200$ (the total number of snapshots) to provide the ensemble average value. It can be seen that the energy contents of the first POD mode slightly higher than, that of the ensemble average value. Consequently, the combination of (a) the flow-pattern equivalency of $\langle\mathrm{V}\rangle$ and $\varphi_{1}^{V}$ and (b) the approximate equivalency of the energy content led to the conclusion that the first POD mode $\varphi_{1}^{V}$ was an excellent estimate of, but not identical to, the ensemble average $\langle V\rangle$. 


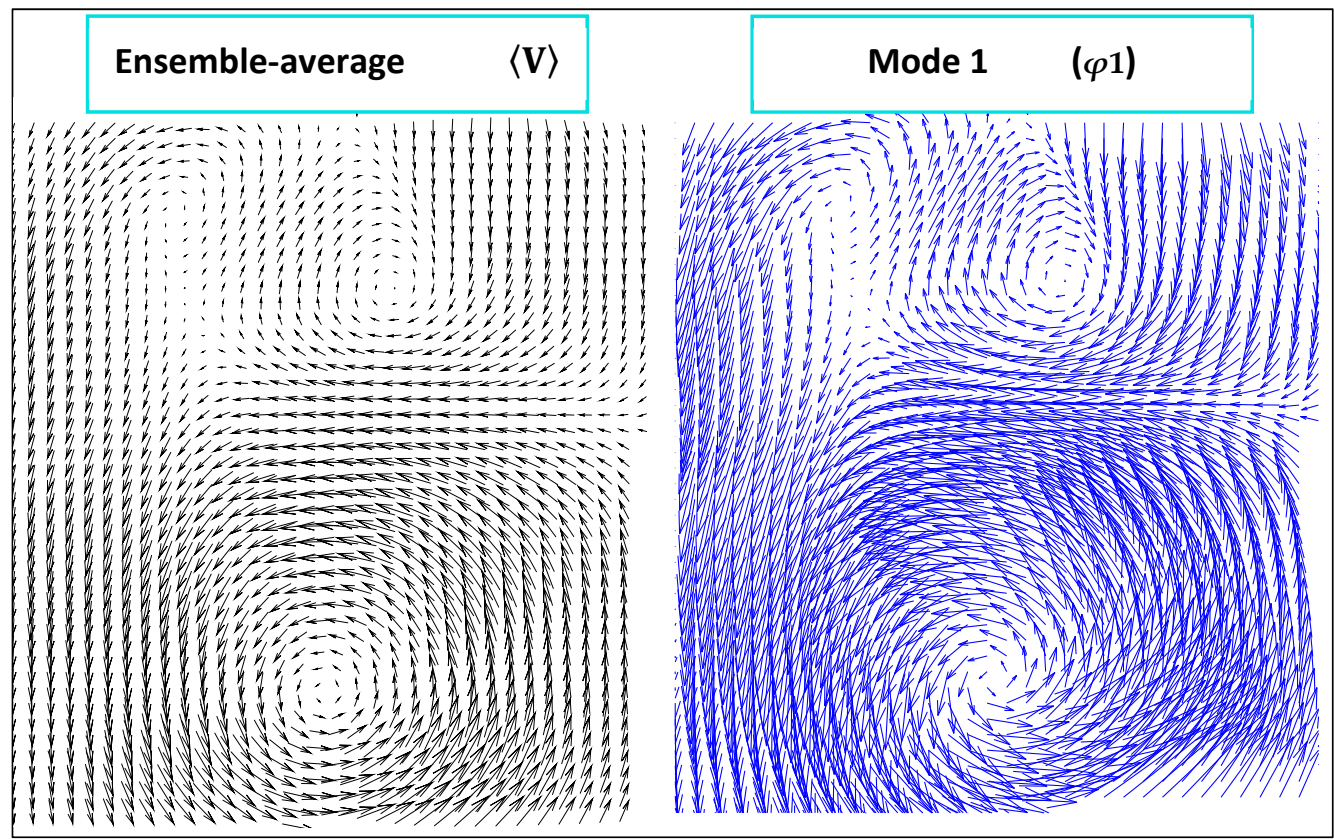

Figure 9. Ensemble average $\langle\mathrm{V}\rangle$ and mode $1(\varphi 1)$ spatial structures without subtracting the mean prior performing POD.

Table 1. Kinetic energy comparison of POD and Reynolds decomposition with and without subtracting the ensemble average.

\begin{tabular}{clccc}
\hline & & $\boldsymbol{K} E$ & $\mathbf{\%}\langle\boldsymbol{V}\rangle$ & $\mathbf{\%} \mathbf{~ r m s}$ \\
\hline Reynold decomposition & $\langle K E\rangle$ & $60,442.3$ & 100 & \\
& $K E_{r m s}$ & $27,944.62$ & & 100 \\
\hline \multirow{2}{*}{ POD on original velocity } & $K E_{\text {total }} / K$ & 88376.4 & & \\
& $K E_{1} / K$ & $60,692.75$ & 100.3 & \\
& $K E_{2} / K$ & 2818.4 & & \\
& $K E_{2-200} / K$ & $27,683.65$ & \\
& $k e_{1}$ & 0.69 & \\
\hline \multirow{2}{*}{ POD after subtracting the ensemble average } & $K E_{\text {total }} / K$ & $27,944.62$ & \\
& $K E_{1} / K$ & 2821.961 & \\
& $k e_{1}$ & 0.1 & \\
\hline
\end{tabular}

\subsubsection{POD and RANS Turbulence}

From the conclusions of the previous section, it was reasonable to deduce that the modes from 2 to $K(K=200)$ contained an estimate of the kinetic energy of the Reynolds-Averaged Navier-Stokes (RANS) turbulence, $K E_{r m s}$. Accordingly, subtracting the ensemble average from the instantaneous velocity vector fields (snapshots) prior to performing the POD, would, by definition, contain only the RANS turbulence. Thus, one can expect that, the flow pattern of $\varphi_{m}^{V-\langle\mathrm{V}\rangle}$ and $\varphi_{m+1}^{V}$ will be identical in case of the flow patterns of the ensemble average and mode 1 were identical. Figure 10 shows the spatial structures of POD modes with and without subtracting the mean. The visual comparison of the patterns of the modes showed that they were not identically equivalent because of the flow structures of mode 1 was not identical to the flow structures of the ensemble average. Moreover, as expected from energy conservation, Table 1 illustrated that $K E_{\text {total }} / K$ of $\mathrm{V}-\langle\mathrm{V}\rangle$ was almost equal to the RANS turbulence, $K_{r m s}$. Also the $K E_{1} / K$ of $V-\langle V\rangle$ was nearly $10 \%$ of $K E_{\text {total }} / K$ signifying that many POD modes would be essential in order to reconstruct the most energetic flow patterns of the Reynolds turbulence. 


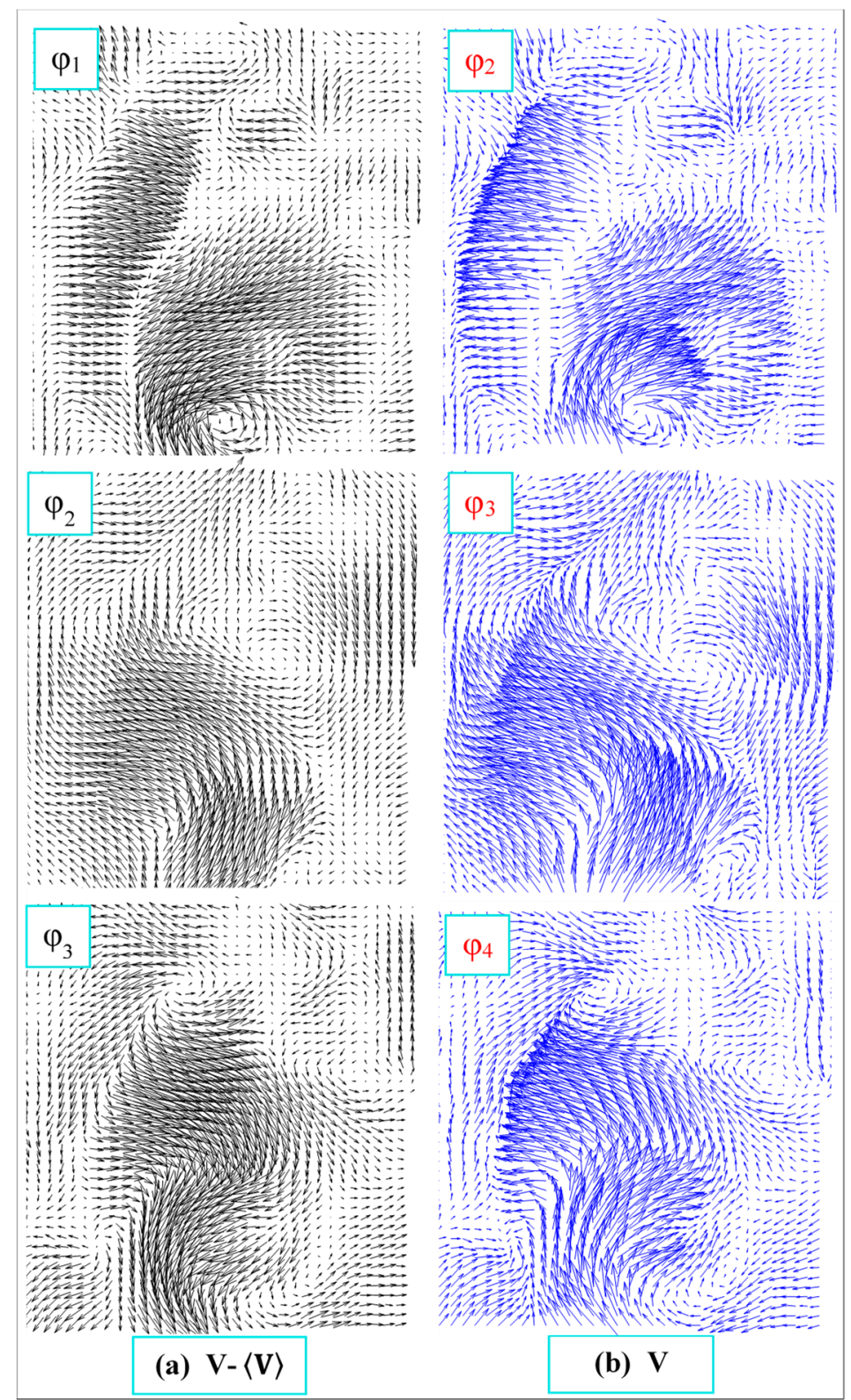

Figure 10. POD modes (a) after subtracting the ensemble average, $\mathrm{V}-\langle\mathrm{V}\rangle$; (b) without subtracting the ensemble average, $\mathrm{V}$.

Figure 11a,b shows the relative energy spectra and absolute energy spectra computed using equations $\left(K E_{m}=\frac{1}{2} \sum_{k=1}^{K}\left(C_{m}^{k}\right)^{2}\right)$ and $\left(K e_{m}=K E_{m} / K E_{\text {total }}\right)$. It can be observed that, the shape of the energy curve either for the energy fraction or absolute energy was quite insensitive to subtracting or not subtracting the ensemble average prior to implementing the POD on the dataset. The biggest change was that the kinetic energy of mode $1, K E_{1}$ for $(\mathrm{V})$ was dominant and contained the main part of the total energy $(70 \%$ in the current example). As a result, there was just an offset in the energy fraction and absolute energy at mode $1(m=1)$ for $\mathrm{V}$ and $\mathrm{V}-\langle V\rangle$ whereas for higher modes $(m>1)$, the shape of the energy curves were identical as shown in the figures. Moreover, the cumulative energy curve shown in Figure 11c reveals that prior to subtracting the mean, mode $1,\left(\varphi_{1}^{V}\right)$, contained $70 \%$ of the energy whereas after subtracting the mean over 30 modes were required to capture $70 \%$ of the total kinetic energy $\left(K E_{\text {total }}\right)$ in case of $\mathrm{V}-\langle V\rangle$. 

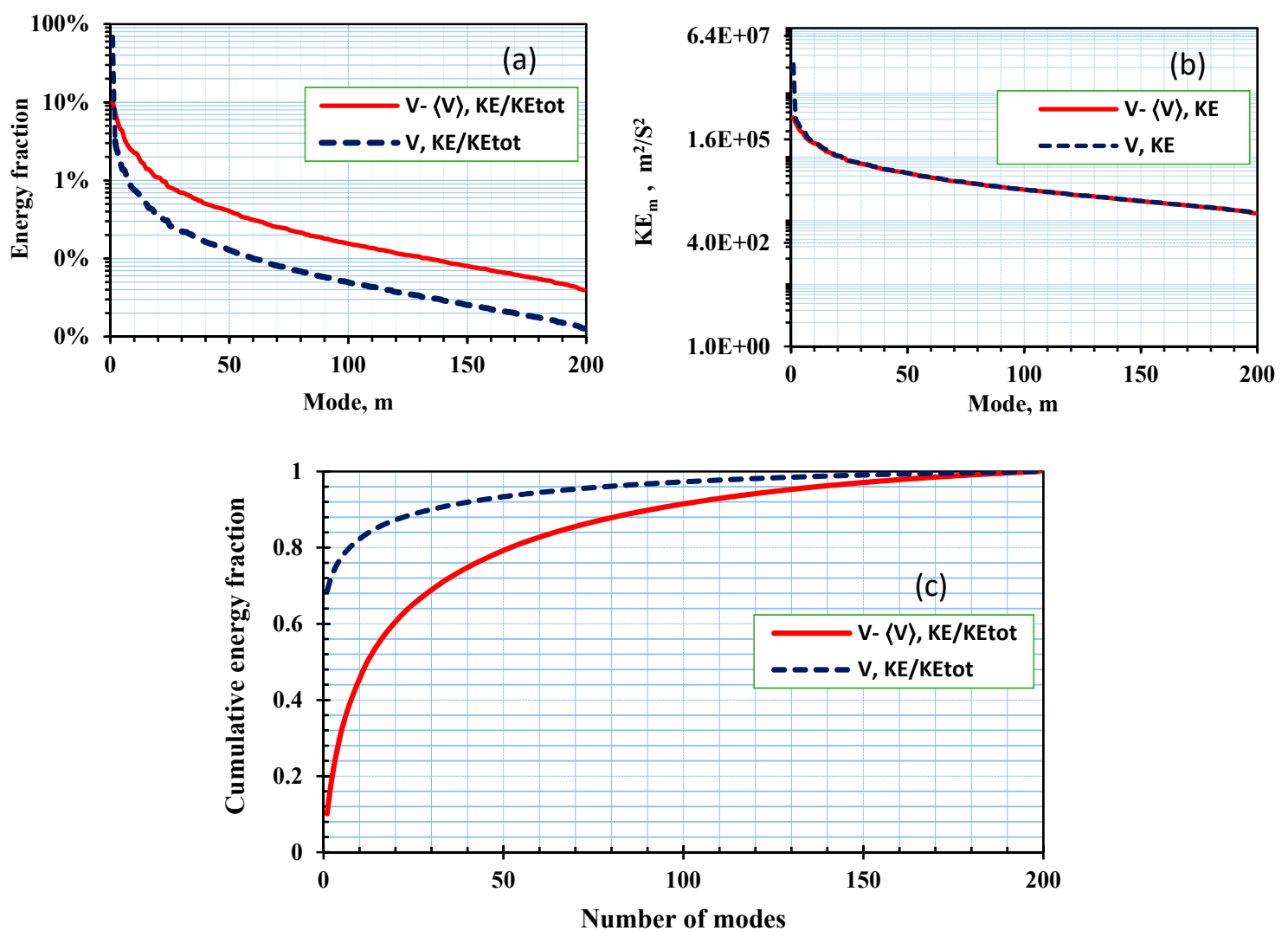

Figure 11. Energy distribution curves for V and V- $\langle V\rangle$ cases. (a) Energy-fraction; (b) Absolute energy, (c) Cumulative energy.

\subsection{POD Analysis of Measured in-Cylinder Engine Flows}

\subsubsection{Evaluation of the Effect of Valve Lift Using Phase-Invariant POD}

A large dataset of 5000 velocity vector fields (snapshots) with the same grid size (same number of vectors) from different valve lifts from $1 \mathrm{~mm}$ to $10 \mathrm{~mm}$ (500 snapshots per valve lift) were arranged to be in one input file and analyzed by using phase-invariant POD to extract the most energetic and organized coherent structures. In this manner, the POD modes could be used to describe the flow structures at any valve lift. While their corresponding coefficients could be used to demonstrate the flow evolution.

- Ensemble Average velocity vector fields

Figure 12 depicts the ensemble average velocity vector fields at different valve lifts. It can be noticed from the figure that, the air flow coming into the cylinder through the intake valves formed two intake air jets, right side (intake side) and the left side (exhaust side). At low valve lifts nearly until $4 \mathrm{~mm}$ lift, the flow was highly restricted on the right side of the intake valves, therefore the right side air jet had a higher velocity than the left side jet. At about valve lift $5 \mathrm{~mm}$, there was a symmetrical velocity distribution between the two intake air jets. Then with increasing the valve lift more, a significant amount of air flow was directed toward the exhaust side and interacted with the left cylinder wall then with the flat piston in the bottom of the cylinder forming a counter clock wise tumble motion. This strong tumble motion dominated the entire cylinder at valve lift $10 \mathrm{~mm}$. 


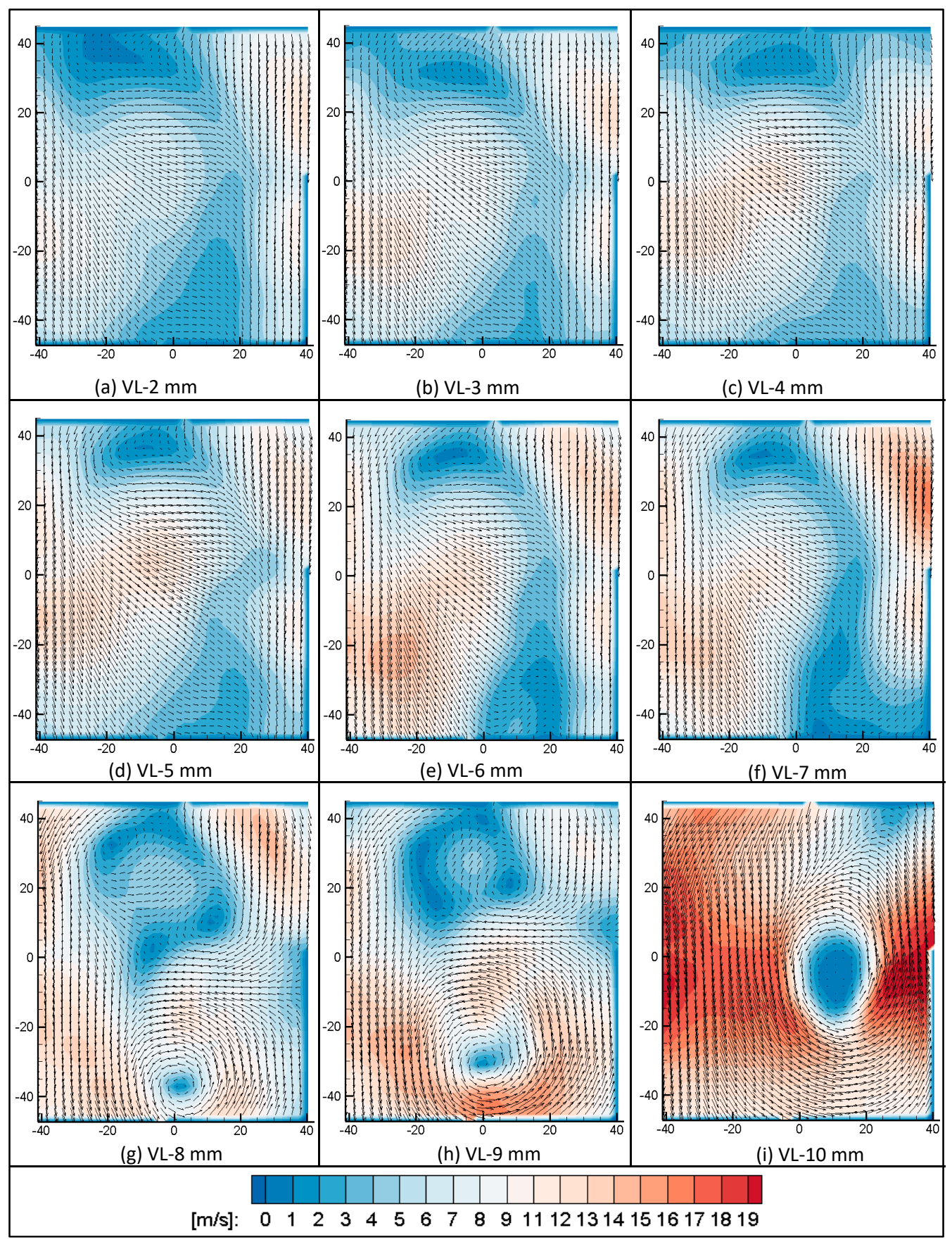

Figure 12. Velocity ensemble average at different valve lifts.

\section{- POD Energy Spectra}

Figure 13 presents the energy fraction captured by the first 20 POD modes to determine the contribution of each mode to the original flow field. It can be seen that the first three modes together extracted about $74 \%$ of the total kinetic energy from all 5000 input velocity vector fields. First mode alone contained $44.5 \%$ of the total energy and second mode contained $18.2 \%$. As shown in the energy graph, the energy level decreased at higher order modes indicating the presence of small scale structures. These small flow structures might not contribute much in the dynamics of the flow. Subsequently, only the interpretation of the first three POD modes flow patterns is introduced in this section. 


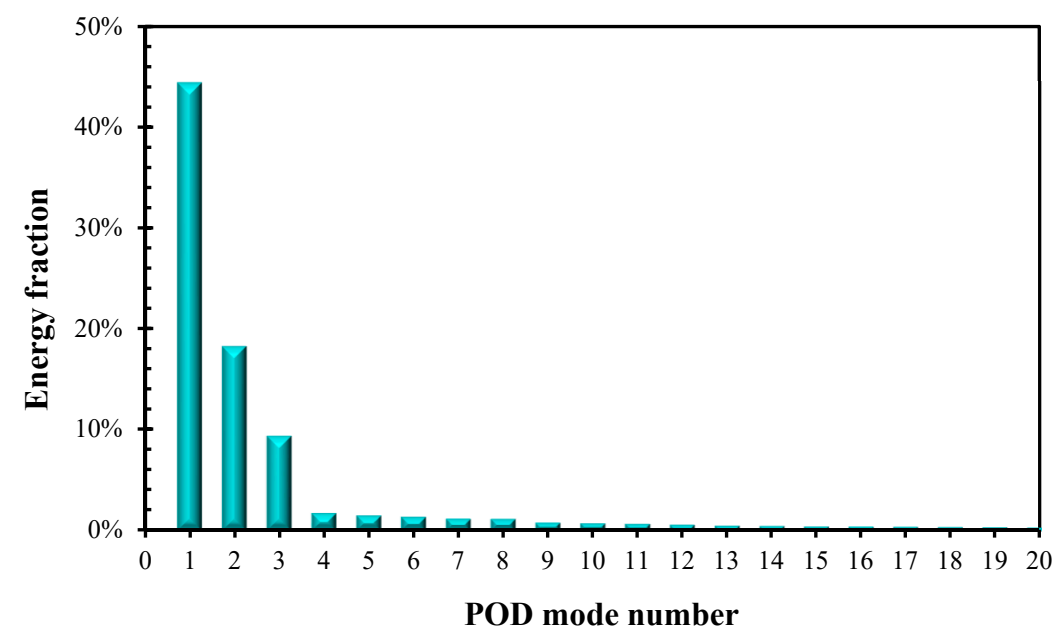

Figure 13. Energy fraction captured by first 20 Proper Orthogonal Decomposition (POD) modes.

\section{- POD Modes Structures and their Corresponding Coefficients}

Figure 14a shows the flow patterns of the first POD mode and its corresponding coefficients at different valve lifts. Each POD mode contained a particular physical flow structure, while the magnitude of the time-dependent coefficients for a specific POD mode illustrated the extent of the dominance for every snapshot. The first mode captured the two intake air jets coming into the cylinder through the right and left sides of the intake valves, as emphasized by the arrows. It can be also noticed from the figure, the difference in intake jet intensity between the right and left sides and how the left side was more dominant. The left intake jet was stronger than the right one. This was attributed to the fact that, the large scale tumbling motion is generally achieved by restricting the flow rate on the outer side of the intake valves (right side) and using straight ports oriented in such a way that most of the annular jet exiting the intake valves is directed towards the exhaust side. Two observations were extracted from Figure 14b. First, positive values of all coefficients were an indication that the flow structures captured by this mode were in the same direction as the original velocity vector fields. Second, when comparing the coefficients at different valve lifts, it was clear that the coefficients increased with increasing the valve lift reaching their maximum values at valve lift $7 \mathrm{~mm}$ then started to decrease again at higher valve lifts. This was expected as the intensity of the intake jet increased with increasing the valve lift. The reductions in coefficients at high valve lifts 8,9 and $10 \mathrm{~mm}$ were attributed to the formation of tumble motion and these valve lifts no longer possessed these jet-like structures much. Figure 15a displays the flow patterns of the second POD mode and its corresponding coefficients at different valve lifts. The first POD mode captured a strong, counter-clockwise, rotating coherent structure in the lower half of the cylinder, indicating a strong positive tumble motion, as highlighted by the dashed circle. This was reasonable as at high valve lifts the left side jet interacted with the left cylinder wall then the flat piston in the bottom of the cylinder leading finally to the formation of tumble motion. Moreover, it is clear from Figure $15 b$ that the high valve lifts 9 and $10 \mathrm{~mm}$ were the only contributors to this flow structures. On the other hand, the values of the coefficients remained zero for the other valve lifts indicating that these valve lifts did not possess this rotating flow structures. Figure 16a shows the flow patterns of the third POD mode and its coefficients at different valve lifts. Mode 3 mainly extracted vortical structures in the lower and upper half of the cylinder. When revising the ensemble average velocity vector fields presented before, one could notice that these flow patterns were found only at valve lifts 8,9 and $10 \mathrm{~mm}$ which was confirmed here by the values of coefficients at these valve lifts. 

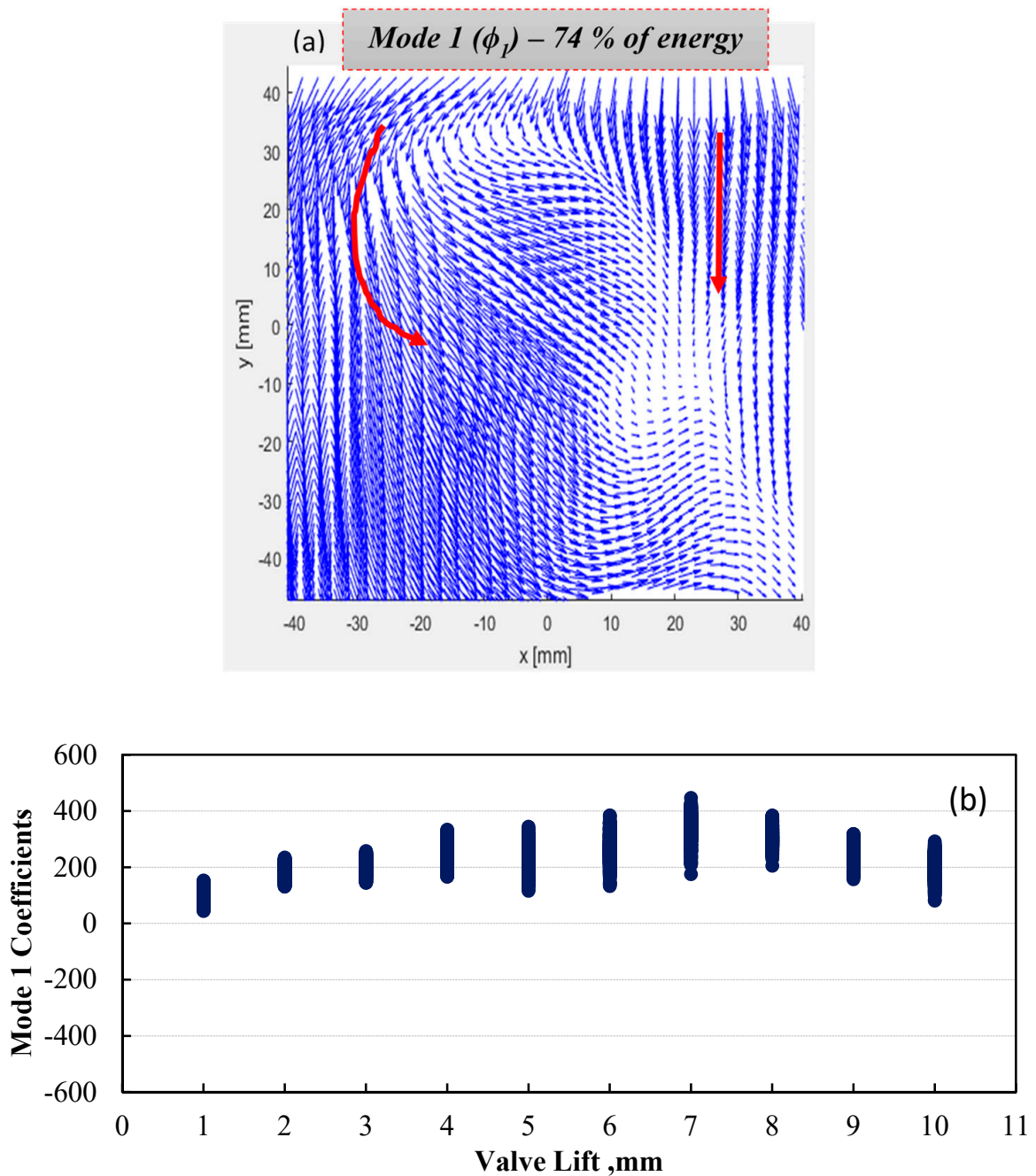

Figure 14. Flow patterns of the first POD mode (a) structures of modes 1 ; (b) mode 1 corresponding coefficients at different valve lifts.

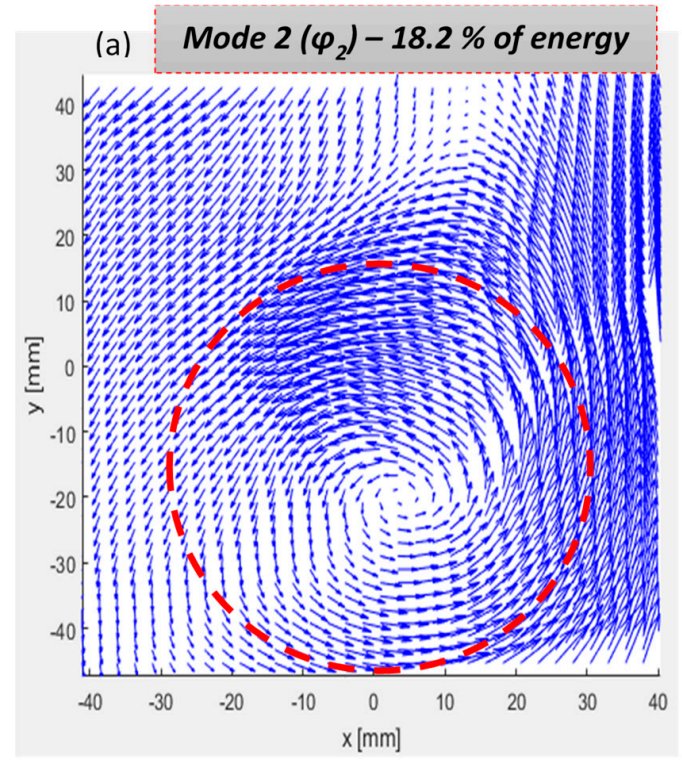

Figure 15. Cont. 


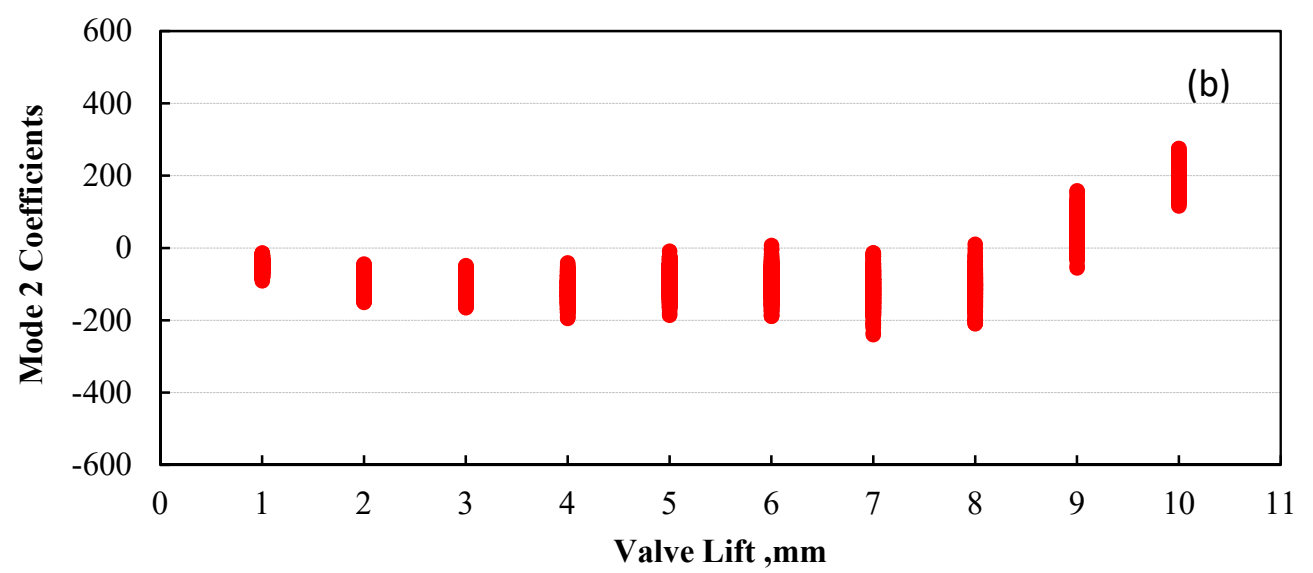

Figure 15. Flow patterns of the second POD mode (a) structures of modes 2; (b) mode 2 corresponding coefficients at different valve lifts.
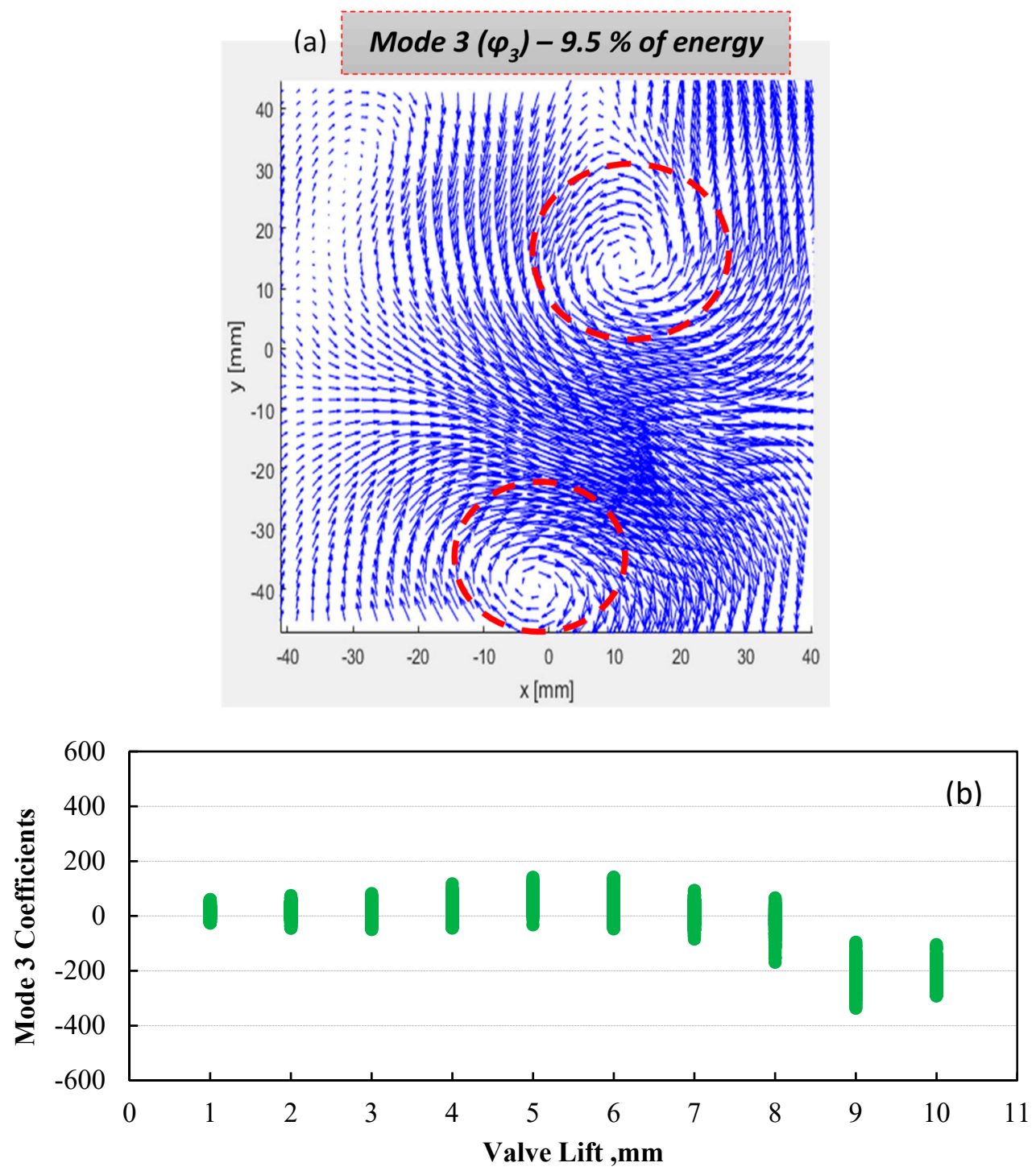

Figure 16. Flow patterns of the third POD mode (a) structures of modes 3; (b) mode 3 corresponding coefficients at different valve lifts. 


\subsubsection{Evaluation of the Effect of Pressure Difference Using Phase-Invariant POD}

In order to characterize the influence of pressure difference on flow structures, three data sets at valve lift $9 \mathrm{~mm}$ for three pressure differences 300, 450 and $600 \mathrm{mmH}_{2} \mathrm{O}$ (1000 velocity vector fields from each pressure difference) were combined into a single file to implement phase-invariant POD. Consequently, under this scenario, for these 3000 input snapshots, 3000 phase-invariant POD modes and their corresponding coefficients were constructed accordingly.

- Phase-invariant POD modes spatial patterns and energy fraction

Figure 17 depicts the spatial patterns of the first four phase-invariant POD modes. In order to show to what extent mode 1 was dominant in these different experimental conditions. The average kinetic energy of the first four POD modes were calculated from their corresponding coefficients. It was found that the average kinetic energy contained in mode 1 , mode 2, mode 3 and mode 4 were $378,008,4884,3892$ and $3529 \mathrm{~m}^{2} / \mathrm{s}^{2}$, respectively. This established one important feature of the effect of pressure difference variation on the in-cylinder flow structures. Even though the input snapshots were collected from different cases of pressure differences, mode 1 only was able to extract more than $85 \%$ of total energy, this quantify the degree of similarity between these datasets. This is consistent with the work of both Krishna [31] and El-Adawy [28]. They illustrated that that the effect of engine speed and subsequently the effect of the pressure difference on the flow structure was negligible compared to the effect of valve lift. This was confirmed in the energy fraction curve shown in Figure 18. This was also consistent with the fact that, the image structures with repeated patterns could be placed in one mode and the other modes were arranged with decreasing order of kinetic energy [15].

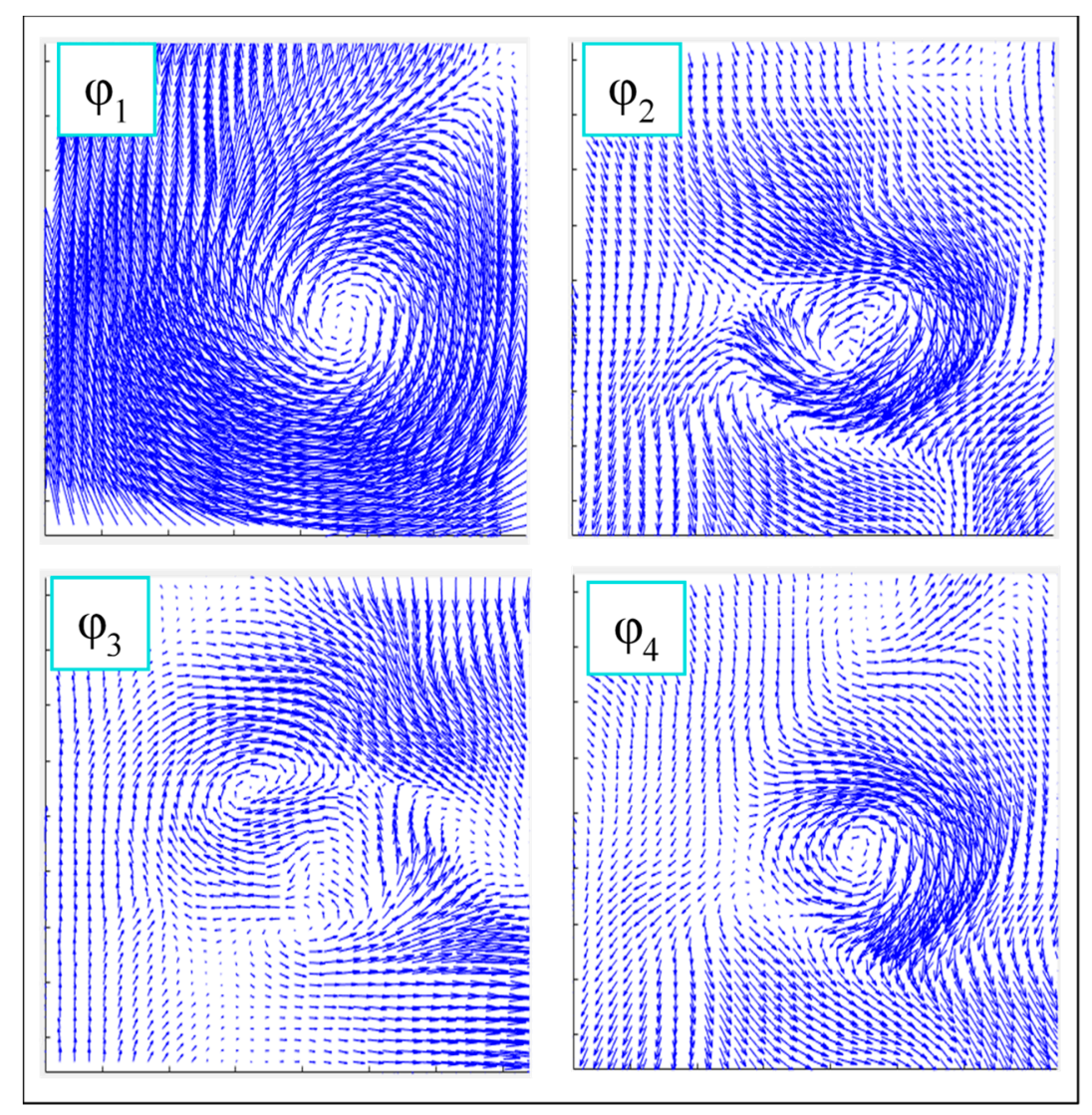

Figure 17. The first four phase-invariant POD modes at valve lift $9 \mathrm{~mm}$. 


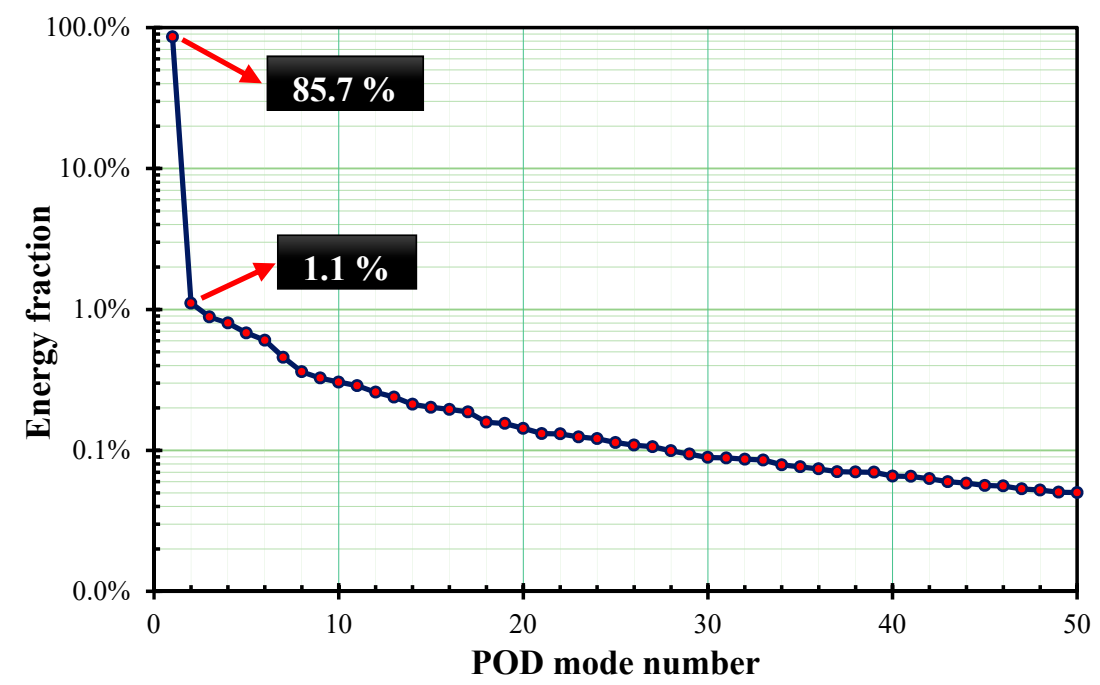

Figure 18. The energy fraction captured by the first 50 phase invariant POD modes at valve lift $9 \mathrm{~mm}$.

Figure 19 depicts the coefficients associated with the first three phase-invariant POD modes versus all 3000 input snapshots. Number of observations were extracted from this Figure. First, there was linear fit between mode 1 coefficients with the applied pressure difference. This was confirmed by the higher value of the coefficient of determination $\mathrm{R}^{2}$, shown in Figure 20, which represented the goodness of data fit. Theoretically, a perfect fit was achieved if the value of the coefficient of determination approached unity. In the current case, $\mathrm{R}^{2}$ was 0.9674 showing the strong linearity existing between mode 1 coefficients and pressure difference. This indicated that the tumbling motion intensity increased with increasing the pressure difference. Second, mode 1 coefficients showed high coefficient of variation (COV) at high pressure difference. For instance, the COV of mode 1 coefficients was $4.42 \%$ and $5.38 \%$ for 300 and $600 \mathrm{mmH}_{2} \mathrm{O}$, respectively. This illustrated in quantitative manner that there was larger snapshot to snapshot variation at high pressure difference than lower one. This was attributed to the fact that higher pressure difference resulted in more air came into the cylinder with higher velocities and subsequently more turbulence.

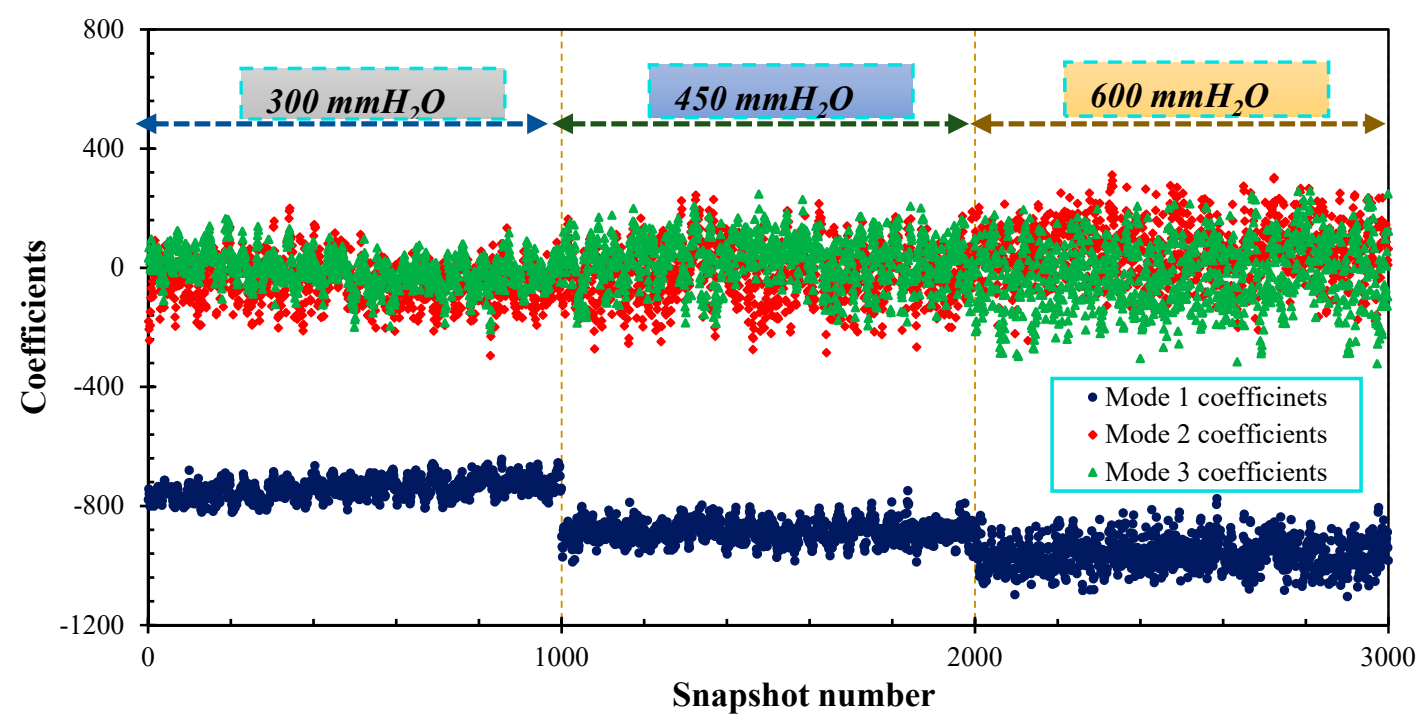

Figure 19. Coefficients associated with the first three phase-invariant POD modes at valve lift $9 \mathrm{~mm}$. 


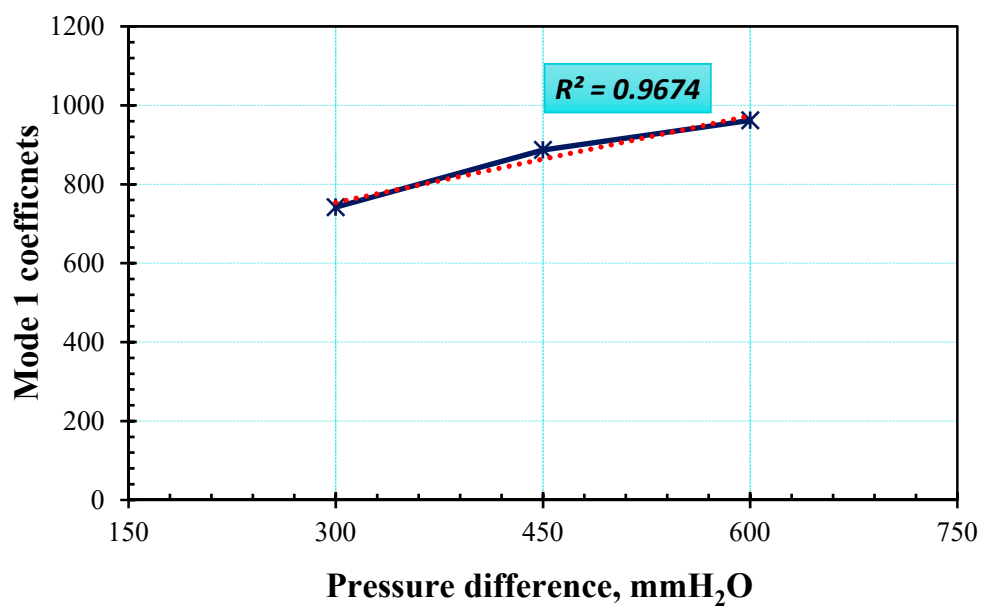

Figure 20. Linear fit between average absolute values of mode 1 coefficients and various pressure differences.

\section{Conclusions}

Firstly, POD was applied to samples of synthetic velocity vector fields with known characteristics with the aim of extracting some important features of POD modes and their corresponding coefficients. Secondly, it is worth recalling that POD is considered as a powerful and quantitative tool for the comparison of experimental datasets. Therefore, POD was used here to compare the in-cylinder flow structures evolution and variation at different valve lifts and different pressure differences as well. The main conclusions can be summarized as follows:

- For repeatable flow patterns, only the first mode is sufficient to reconstruct the physical flow structures and its corresponding coefficients will be identical for all snapshots.

- In the case of implementing the POD without subtracting the ensemble mean, the first mode was an excellent estimate of the flow pattern of the ensemble average.

- Subtracting the ensemble average from the instantaneous velocity vector fields (snapshots) prior to performing the POD, would, by definition, contain only the RANS turbulence.

- For the effect of valve lift, the first mode captured the two intake air jets coming into the cylinder through the right and left sides of the intake valves and showed that how the right side jet was weaker than the left one because of the concept of tumbling motion generation. While the second and third modes captured the tumble motion generated at high valve lifts.

- For the effect of pressure difference, even though the input snapshots were collected from different cases of pressure differences, mode 1 only was able to extract more than $85 \%$ of total energy, this quantified the degree of similarity between these datasets and illustrated the effect of pressure difference on in-cylinder flow structures. Regardless the applied pressure difference, the in-cylinder flow patterns did not change, however, the level of the turbulence kinetic energy increased as the pressure difference increased. This was very clear from the values of mode 1 coefficients.

Author Contributions: The investigation was leaded and supervised by M.R.H. and A.R.A.A. The experimental work and analysis and most illustrations were conducted by M.E.A., I.K.A., M.A.I., M.E.B., M.B.B., F. and E.Z.Z.A. contributed in data analysis and the preparation of the experimental set up.

Acknowledgments: The current work was carried out in a collaboration between Universiti Teknologi PETRONAS (UTP) through the Centre for Automotive Research and Energy Management (CAREM) (PRGS/1/2017/TK07/UTP/01/1) and Ricardo UK.

Conflicts of Interest: The authors declare no conflict of interest. 


\section{References}

1. Porpatham, E.; Ramesh, A.; Nagalingam, B. Effect of swirl on the performance and combustion of a biogas fuelled spark ignition engine. Energy Convers. Manag. 2013, 76, 463-471. [CrossRef]

2. Lee, K.; Bae, C.; Kang, K. The effects of tumble and swirl flows on flame propagation in a four-valve SI engine. Appl. Therm. Eng. 2007, 27, 2122-2130. [CrossRef]

3. Mittal, M.; Hung, D.L.; Zhu, G.; Schock, H. High-Speed Flow and Combustion Visualization to Study the Effects of Charge Motion Control on Fuel Spray Development and Combustion Inside a Direct-Injection Spark-Ignition Engine. SAE Int. J. Engines 2011, 4, 1469-1480. [CrossRef]

4. El-Adawy, M.; Heikal, M.R.; Aziz, A.R.A.; Siddiqui, M.I.; Munir, S. Characterization of the Inlet Port Flow under Steady-State Conditions Using PIV and POD. Energies 2017, 10, 1950. [CrossRef]

5. El-Adawy, M.; Heikal, M.; Aziz, A.R.A.; Siddiqui, M.; Wahhab, H.A.A. Experimental study on an IC engine in-cylinder flow using different steady-state flow benches. Alex. Eng. J. 2017, 56, 727-736. [CrossRef]

6. Zhang, Z.; Zhang, H.; Wang, T.; Jia, M. Effects of tumble combined with EGR (exhaust gas recirculation) on the combustion and emissions in a spark ignition engine at part loads. Energy 2014, 65, 18-24. [CrossRef]

7. Bari, S.; Saad, I. CFD modelling of the effect of guide vane swirl and tumble device to generate better in-cylinder air flow in a CI engine fuelled by biodiesel. Comput. Fluids 2013, 84, 262-269. [CrossRef]

8. Brusiani, F.; Falfari, S.; Cazzoli, G. Tumble motion generation in small gasoline engines: A new methodological approach for the analysis of the influence of the intake duct geometrical parameters. Energy Procedia 2014, 45, 997-1006. [CrossRef]

9. Buhl, S.; Gleiss, F.; Köhler, M.; Hartmann, F.; Messig, D.; Brücker, C.; Hasse, C. A combined numerical and experimental study of the $3 \mathrm{D}$ tumble structure and piston boundary layer development during the intake stroke of a gasoline engine. Flow Turbul. Combust. 2017, 98, 579-600. [CrossRef]

10. Lumley, J.L. The structure of inhomogeneous turbulent flows. In Atmospheric Turbulence and Radio Wave Propagation; Publishing House Nauka: Moscow, Russia, 1967.

11. Zhuang, H.; Hung, D.L. Characterization of the effect of intake air swirl motion on time-resolved in-cylinder flow field using quadruple proper orthogonal decomposition. Energy Convers. Manag. 2016, 108, 366-376. [CrossRef]

12. Zhuang, H.; Hung, D.L.; Yang, J.; Tian, S. Investigation of Swirl Ratio Impact on In-Cylinder Flow in an SIDI Optical Engine. J. Eng. Gas Turbines Power 2016, 138, 081505. [CrossRef]

13. Voisine, M.; Thomas, L.; Borée, J.; Rey, P. Spatio-temporal structure and cycle to cycle variations of an in-cylinder tumbling flow. Exp. Fluids 2011, 50, 1393-1407. [CrossRef]

14. Chen, H.; Reuss, D.L.; Sick, V. Analysis of misfires in a direct injection engine using proper orthogonal decomposition. Exp. Fluids 2011, 51, 1139. [CrossRef]

15. Chen, H.; Hung, D.; Xu, M.; Zhong, J. Analyzing the cycle-to-cycle variations of pulsing spray characteristics by means of the proper orthogonal decomposition. At. Sprays 2013, 23, 632-641. [CrossRef]

16. Bizon, K.; Continillo, G.; Leistner, K.; Mancaruso, E.; Vaglieco, B. POD-based analysis of cycle-to-cycle variations in an optically accessible diesel engine. Proc. Combust. Inst. 2009, 32, 2809-2816. [CrossRef]

17. Di Mare, F.; Knappstein, R. Statistical analysis of the flow characteristics and cyclic variability using Proper Orthogonal Decomposition of highly resolved LES in internal combustion engines. Comput. Fluids 2014, 105, 101-112. [CrossRef]

18. Druault, P.; Guibert, P.; Alizon, F. Use of proper orthogonal decomposition for time interpolation from PIV data. Exp. Fluids 2005, 39, 1009-1023. [CrossRef]

19. Chen, H.; Xu, M.; Hung, D.L.; Yang, J.; Zhuang, H. Development of a POD-Based Analysis Approach for Quantitative Comparison of Spray Structure Variations in a Spark-Ignition Direct-Injection Engine; SAE Technical Paper 0148-7191; SAE International: Warrendale, MI, USA, 2013.

20. Qin, W.; Hung, D.L.; Xu, M. Investigation of the temporal evolution and spatial variation of in-cylinder engine fuel spray characteristics. Energy Convers. Manag. 2015, 98, 430-439. [CrossRef]

21. Chen, H.; Reuss, D.L.; Sick, V. On the use and interpretation of proper orthogonal decomposition of in-cylinder engine flows. Meas. Sci. Technol. 2012, 23, 085302. [CrossRef]

22. Wang, T.; Li, W.; Jia, M.; Liu, D.; Qin, W.; Zhang, X. Large-eddy simulation of in-cylinder flow in a DISI engine with charge motion control valve: Proper orthogonal decomposition analysis and cyclic variation. Appl. Therm. Eng. 2015, 75, 561-574. [CrossRef] 
23. Kapitza, L.; Imberdis, O.; Bensler, H.P.; Willand, J.; Thevenin, D. An experimental analysis of the turbulent structures generated by the intake port of a DISI-engine Exp. Fluids 2010, 48, 265-280.

24. Chen, H.; Reuss, D.L.; Hung, D.L.; Sick, V. A practical guide for using proper orthogonal decomposition in engine research. Int. J. Engine Res. 2013, 14, 307-319. [CrossRef]

25. Fabrizio, V.; Maddalena, L. Stereoscopic particle image velocimetry measurements of supersonic, turbulent, and interacting streamwise vortices: Challenges and application. Prog. Aerosp. Sci. 2014, 66, 1-16.

26. Jean, R.; Vernet, J.A.; Lindgren, B.; Alfredsson, P.H. A study using PIV of the intake flow in a diesel engine cylinder. Int. J. Heat Fluid Flow 2016, 62, 56-67.

27. Liu, Z.; Zheng, Y.; Jia, L.; Jiao, J.; Zhang, Q. Stereoscopic PIV studies on the swirling flow structure in a gas cyclone. Chem. Eng. Sci. 2006, 61, 4252-4261. [CrossRef]

28. El Adawy, M.; Heikal, M.R.; Aziz, A.R.A.; Munir, S.; Siddiqui, M.I. Effect of Boost Pressure on the In-Cylinder Tumble-Motion of GDI Engine under Steady-State Conditions using Stereoscopic-PIV. J. Appl. Fluid Mech. 2018, 11, 733-742.

29. Rabault, J. PIV Investigation of the Intake Flow in a Parallel Valves Diesel Engine Cylinder; Royal Institute of Technology KTH Mechanics: Stockholm, Sweden, 2015.

30. Sirovich, L. Turbulence and the dynamics of coherent structures. I. Coherent structures. Q. Appl. Math. 1987, 45, 561-571. [CrossRef]

31. Krishna, B.M.; Mallikarjuna, J. Effect of engine speed on in-cylinder tumble flows in a motored internal combustion engine-An experimental investigation using particle image velocimetry. J. Appl. Fluid Mech. 2011, 4, 1-14.

(c) 2018 by the authors. Licensee MDPI, Basel, Switzerland. This article is an open access article distributed under the terms and conditions of the Creative Commons Attribution (CC BY) license (http:/ / creativecommons.org/licenses/by/4.0/). 